\title{
A Fast Direct Search Algorithm for Contact Detection of Convex Polygonal or
}

\author{
Polyhedral Particles
}

Fei Zheng ${ }^{\text {a,b,c }}$, Yu-Yong Jiao ${ }^{a^{*}}$, Michael Gardner ${ }^{\mathrm{c}}$, Nicholas Sitar

\begin{abstract}
A Fast Direct Search (FDS) algorithm is presented to increase the efficiency of contact detection for convex polygonal and polyhedral particles. All contact types are detected using only a small subset of these contact types: vertex-to-edge for polygons while vertex-to-face and edge-to-edge for polyhedra. First, an initial contact list is generated. Then in subsequent steps the contact list is updated by checking only local boundaries of the blocks and their separation. An exclusion algorithm is applied to avoid unnecessary examination for particles that are near but not-in-contact. The benchmark tests show that the FDS produces significant speed-up in various cases.
\end{abstract}

Keywords: Contact detection; convex particles; valid entrance; initial detection; updated detection 


\section{Introduction}

23 Contact analysis is a necessary part of any computational method dealing with

24 interaction between independent particles, such as in the discrete element method 25 (DEM) and discontinuous deformation analysis (DDA). Contact detection comprises a 26 significant portion of the computational effort in these types of analyses and therefore an accurate and efficient contact detection algorithm is essential. The aim of the contact detection phase is to provide possible contact types, contact points, contact normal directions and contact modes for all potential contact elements. Focusing on contacts between two particles, the particle shape becomes an important factor

31 affecting computation accuracy and efficiency. In rock engineering, rock masses typically consist of densely packed polyhedral blocks and contact detection between polyhedral blocks is more complicated than between spheres, which are common in other applications. Moreover, the efficiency of contact detection between convex polyhedra is much higher than that for concave polyhedra, owing to the utilization of convex properties in the detection algorithm. This paper focuses on improving the efficiency and accuracy of contact detection for convex polyhedra.

From a geometrical perspective, convex polygons and convex polyhedrons have distinct properties which can be used in geometry representation and contact detection. For the geometric representation, two main methods are used. First, the point set of a two-dimensional (2-D) convex polygon or a three-dimensional (3-D) convex polyhedron can be algebraically represented by the intersection of several inequalities. 
The linear programming method [1] uses these expressions as a basis. Second, a boundary-representative method for polygons or polyhedra can be used. Boundaries of a polygon consist of vertices and edges while boundaries of a polyhedron consist of vertices, edges and faces.

Several convex polyhedron properties can be used in contact detection. First, a virtual infinite, rigid plane, named the common plane [2], can be found to separate two polygons or two polyhedra. This common plane can be considered as a reference plane to quickly compute the contact point and contact normal direction. Second, if a point is inside a polyhedron (polygon), this point must be inside all the half-spaces that form the polyhedron (polygon). As a result, if a point is outside any half-space forming the polyhedron (polygon), it is outside the polyhedron (polygon). This property is quite useful in being able to exclude vertices that are not in contact. Third, all 2-D planar angles, 3-D solid angles and dihedrals forming boundaries of convex polyhedrons are convex. Fourth, contacts of two convex polygons fall into the following types: vertex-to-vertex ( $v-v)$, vertex-to-edge ( $v-e)$ and parallel edge-to-edge (parallel e-e), whereas contacts of two convex polyhedra consist of: vertex-to-vertex $(v-v)$, vertex-to-edge (v-e), vertex-to-face (v-f), crossing edge-to-edge (crossing e-e), parallel edge-to-edge (parallel e-e), edge-to-face (e-f) and face-to-face (f-f).

1 Numerous contact detection algorithms have been presented to improve the efficiency of detecting contacts between convex polyhedra. One approach is the common plane algorithm [2]. The common plane simplifies the contact detection process by testing 

blocks. This approach was further optimized to produce the fast common plane (FCP) algorithm [3] and the shortest link method (SLM) [4]. Another genre is referred to as the direct search or improved direct search algorithms [8-10]. Other methods include the approaching face method [5], an improved approaching face algorithm [6], vertex-to-face searching algorithm [7] and multi-shell cover method [11]. Boon et al. [1] generalize contact between convex polyhedral particles as a convex optimization problem and used a linear program method to solve it.

Most recently, Shi [12] proved that contact between two convex polyhedra can be

73 simplified to contact of a reference point and an entrance convex polyhedron. The

74 boundary of the entrance polyhedron consists of contact covers ( $\mathrm{v}-\mathrm{v}, \mathrm{v}-\mathrm{e}, \mathrm{v}-\mathrm{f}$ and crossing e-e), and the position relationship of the reference point and contact covers determines the contact point, contact normal direction and contact mode. In this paper,

77 we build on the theoretical work of Shi [12] and present the fast direct search (FDS)

78 algorithm to improve the efficiency and accuracy of the contact detection. The FDS

79 algorithm is applied in DDA to do discontinuous computation, two basic types ( $\mathrm{v}-\mathrm{v}$ 80 and v-e) are used in 2-D case and four basic contact types (v-v, v-e, v-f and crossing 81 e-e) are used in 3-D case for contact force computation.

\section{The fast direct search algorithm (FDS)}

83 The contact detection procedure consists of two search phases: neighbor search and 84 delicate search. The neighbor search algorithm is based on the cell mapping method 
85 [2], but can be optimized using other neighbor search algorithms - such as NBS [13],

86 DESS [14] or CGrid [15] - depending on the application. The fast direct search (FDS)

87 algorithm is specifically concerned with the delicate search during which contact

88 points, normals and modes are established.

89 FDS aims to obtain contact geometry information for convex polygonal or polyhedral

90 particles more efficiently. In order to obtain higher efficiency, the FDS algorithm is

91 executed in two phases. First, initial contact detection is executed and then updated

92 contact detection is initiated when necessary. The initial contact detection phase forms

93 a list of contact types for a new block geometry configuration, while the updated

94 contact detection phase updates the contact information in this list starting from the

95 contact detection results in the previous iteration.

96 Herein the basic concepts used in FDS are first introduced and then a detailed

97 discussion of the contact detection process, data structures used and associated 98 computational cost is presented.

$99 \quad 2.1$ Basic concepts used in contact detection

$100 \quad$ 2.1.1 Valid entrance concept

101 Valid entrance is defined as a physical no-overlap status of potential contact pairs,

102 similar to the concept of first entrance developed by Shi [12] [16]. Valid entrance

103 means that two potential contact objects should not have an overlapping area if their

104 potential contact points are superimposed. In this way, a valid entrance check is used

105 as a criterion in judging basic contact pairs. No overlap between two contact objects, 
106 from another perspective, is the same as the concept of a continuous face that

107 separates these two objects. Here for convex objects, a plane separating the two

108 objects can be found if they do not overlap.

109 A 2-D vertex-to-edge, v-e, entrance is valid if both edge vectors of the vertex angle

110 point out of the half-space formed by the edge. As shown in Fig.1, vertex $v_{0}$ and edge

$111 e_{0} e_{1}$ is a valid entrance pair because both edge vector $v_{0} v_{1}$ and $v_{0} v_{2}$ point out of

112 half-space surrounded by edge $e_{0} e_{1}$. In 2-D vertex-to-vertex, v(i)-v(j), entrance

113 checking, all edge $\mathrm{e}(i)$ and $\mathrm{e}(j)$ joint to vertex $\mathrm{v}(i)$ and $\mathrm{v}(j)$ are considered. $\mathrm{V}(i)-\mathrm{v}(j)$

114 entrance checking is subdivided into $\mathrm{v}(i)-\mathrm{e}(j)$ and $\mathrm{e}(i)-\mathrm{v}(j)$ entrance checking. If any

$115 \mathrm{v}(i)-\mathrm{e}(j)$ or $\mathrm{e}(i)-\mathrm{v}(j)$ entrance is valid, $\mathrm{v}(i)-\mathrm{v}(j)$ entrance is valid. Then half-space outer

116 normals of all valid $\mathrm{v}(i)-\mathrm{e}(j)$ or $\mathrm{e}(i)-\mathrm{v}(j)$ entrances are stored in a list of potential

117 contact normals.

118 In 3-D, vertex-to-face, v-f, or crossing edge-to-edge, e-e, entrance is valid if an

119 infinite plane can be found that separates the vertex angle and the half-space

120 surrounded by the face, or separates the two dihedrals connecting the two edges. For

$121 \mathrm{v}-\mathrm{f}$ entrance, the face can serve as the separating plane, and two steps are used in

122 checking validity of v-f entrance. First, the average value of edge vectors that join the

123 vertex angle is obtained and used to roughly judge if the vertex angle overlaps the

124 half-space formed by the face. If the average vector points into the half-space, overlap

125 take place and the v-f entrance is not valid; otherwise, the second step is conducted.

126 All edge vectors that joint the vertex angle are used to check overlap towards the 
128 face normal is a valid entrance plane normal; otherwise, the v-f entrance is invalid.

129 For a crossing e-e entrance, the normal of a potential separating plane is obtained by

130 the cross product of vectors along the two edges. Then two edge vectors along the

131 edge dihedral faces are obtained for each edge. These vectors are used to judge if the

132 two edges are separated by the contact plane, as shown in Fig.3. If they are, then this

133 e-e pair is a valid entrance and the potential contact plane normal is a valid entrance

134 plane normal; otherwise, it is invalid. In 3-D v(i)-v(j) or $\mathrm{v}(i)-\mathrm{e}(j)$ entrance checking,

135 all edges and faces $\mathrm{e}(i), \mathrm{e}(j), \mathrm{f}(i)$ and $\mathrm{f}(j)$, that joint the vertex angles, and the edge

136 dihedrals are considered. $\mathrm{V}(i)-\mathrm{v}(j)$ or $\mathrm{v}(i)-\mathrm{e}(j)$ entrance checking is subdivided into

$137 \mathrm{v}(i)-\mathrm{f}(j), \mathrm{f}(i)-\mathrm{v}(j)$ and $\mathrm{e}(i)-\mathrm{e}(j)$ entrance checking. If any of these subset entrance is

138 valid, $\mathrm{v}(i)-\mathrm{v}(j)$ entrance is valid. Then half-space outside normals of all valid $\mathrm{v}(i)-\mathrm{f}(j)$,

$139 \mathrm{f}(i)-\mathrm{v}(j)$ and $\mathrm{e}(i)-\mathrm{e}(j)$ entrance are stored in a list of potential contact normals.

\section{$140 \quad$ 2.1.2 Contact patterns}

141 Contacts of 2-D polygons include v-v, v-e and parallel e-e types; however, all these

142 contact types can be described using only the v-e contact type: parallel e-e contacts

143 can be regarded as a combination of v-v and v-e contacts. So, for contact detection of

144 2-D polygons, only v-e pairs are directly examined. Then according to the number of

145 valid v-e contact pairs, the final contact type (v-v and v-e) can be deduced.

146 In 3-D, possible contact types are v-v, v-e, v-f, parallel e-e, crossing e-e, e-f and f-f.

147 Parallel e-e contacts can be regarded as a combination of $v-v$ and v-e contacts. E-f and 
148

149

151

152

153

154

155

$158 \mathrm{v}$-f and crossing e-e) while only needing to directly detect v-f and crossing e-e contact

159 pairs.

\section{$160 \quad$ 2.1.3 Virtual Contact Plane (VCP)}

161 Each contact defines a pair of virtual common planes, defined by a point and a normal.

162 The two VCPs pass through respective contact points defined as follows: (1) the

163 points that give the minimum distance between the two objects before they contact; (2)

164 the points that give the shortest path to separate the penetrating objects when they

165 contact. For example, the contact points of a v-f or v-e contact pair are the vertex and

166 its orthogonal projection point on the face or the edge. The VCP normal of a contact

167 pair is calculated using the valid entrance plane normals obtained during the valid 
entrance check. The normal points out of the polygon or polyhedron and, consequently, each VCP pair has opposite normal vectors, as shown in Fig. 1-3.

170 For a 2-D v-e contact pair, the VCPs are two lines passing the vertex and its

171 orthogonal projection point onto the edge, respectively and they are parallel to the

172 edge, as shown in Fig.1. For a 2-D v-v contact pair, the VCPs pass through the two

173 vertices. A list of potential VCP normals has been established in v-v valid entrance

174 check. The average of the vectors in potential VCP normal list is selected as the VCP

175 normal, as shown in Fig.4. Similarly, for a 3-D contact pair including faces (v-f, e-f

176 and f-f), the VCP normals are parallel to the outer normal of their unique valid

177 entrance faces and point out of the blocks, as shown in Fig.2. For 3-D crossing e-e

178 contact pairs, VCP normals are parallel to the cross product of the two edge vectors,

179 as shown in Fig.3. The determination of the VCP normal for a v-v or v-e contact pairs

180 is executed in two steps. First, a list of potential VCP normals is established in 3-D

$181 \mathrm{~V}-\mathrm{v}$ or v-e entrance checking (chapter 2.1.1). Second, the average of the vectors in the

182 VCP normal list is obtained and serves as the VCP normal for this contact pair. It

183 should be mentioned that if the list of the potential VCP normals is empty, then the

$184 \mathrm{v}-\mathrm{v}$ or v-e pair is not a valid entrance pair.

185 The VCP is applied in two aspects: (1) its normal can be used as the contact normal

186 for its related contact pair; (2) vertices in its search region (defined in chapter 2.1.4)

187 are used to define the nearby vertices, edges or faces for neighbor block pairs in the 188 updated contact detection phase. 


\subsubsection{Exclusion algorithm and contact tolerance}

190 During the neighbor search, some blocks may be regarded as possibly being in contact

191 based on their bounding boxes or spheres. However, during the delicate search it is

192 established that they are actually not in contact. To avoid unnecessary examination for

193 these block pairs, an exclusion algorithm is applied in the FDS using contact tolerance

194 criteria.

195 Empirical tolerance criteria have to be defined for each contact type. In this work we

196 define for a parallel e-e pair is one degree. The minimum distance tolerance $d_{t o l}$ to

197 determine potential contact pairs is set as $d_{\text {tol }}=\max \left\{2 \cdot d_{\max }, 0.05 \cdot d_{\text {small }}\right\}$, while $d_{\max }$

198 refers to the maximal block vertex displacement per time step; $d_{\text {small }}$ refers to the

199 smallest edge length of the block system. If the minimum distance of a checking pair

200 is smaller than $d_{t o l}$, this pair satisfies the distance judging criterion for potential

201 contact pairs.

$202 d_{t o l}$ is also used as the orthogonal distance tolerance to determine if one vertex locates

203 in search regions of a virtual contact plane (VCP) region. If the orthogonal distance

$204 d_{v p}$ between a vertex and a VCP is smaller than $d_{t o l}$, the vertex is in VCP regions. So,

205 the VCP searching region is the space formed by translating VCP from $-d_{t o l}$ to $d_{t o l}$ 206 along its normal.

207 Thus, in the examination of v-e (2-D), v-f and crossing e-e (3-D) pairs, the orthogonal

208 distance $d_{o}$ of these pairs is obtained. If the orthogonal distance $d_{o}$ of a checking pair 209 is larger than $d_{t o l}$, the pair may be too far apart to be in contact during the current time 
210 step. If their mutual entrance status is valid (no penetration) in addition to $d_{o}$ being

211 larger than $d_{t o l}$, then the block pair is regarded as not-in-contact during the current

212 time step and the delicate search process for this block pair is skipped.

\section{$213 \quad 2.2$ Initial contact detection}

214 The initial contact detection considers the new geometry configuration. In 2-D, a

215 thorough v-e search is executed in the initial detection of two convex polygons, as

216 shown in Fig.5. V(i)-e(j) check is first executed, in which all $\mathrm{v}(i)-\mathrm{v}(j)$ and $\mathrm{v}(i)-\mathrm{e}(j)$

217 types are established. Then $\mathrm{e}(i)-\mathrm{v}(j)$ check follows and all $\mathrm{e}(i)-\mathrm{v}(j)$ types are detected 218 out.

219 In 3-D, $\mathrm{v}(i)-\mathrm{f}(j), \mathrm{f}(i)-\mathrm{v}(j)$ and $\mathrm{e}(i)-\mathrm{e}(j)$ contact pairs are checked one by one to find the 220 unique contact type for the two convex polyhedra, as shown on the flow chart in Fig.6.

221 In the $\mathrm{v}(i)-\mathrm{f}(j)$ check, all $\mathrm{v}(i)-\mathrm{v}(j), \mathrm{v}(i)-\mathrm{e}(j)$ and $\mathrm{v}(i)-\mathrm{f}(j)$ types are established. In 222 subsequent $\mathrm{f}(i)-\mathrm{v}(j)$ check, all $\mathrm{e}(i)-\mathrm{v}(j)$ and $\mathrm{f}(i)-\mathrm{v}(j)$ are contacts are detected and, 223 finally, all $\mathrm{e}(i)-\mathrm{e}(j)$ contacts are identified.

\section{$224 \quad$ 2.2.1 Vertex-to-face check}

225 All 3-D v(i)-v(j), $\mathrm{v}(i)-\mathrm{e}(j), \mathrm{v}(i)-\mathrm{f}(j), \mathrm{e}(i)-\mathrm{v}(j)$ and $\mathrm{f}(i)-\mathrm{v}(j)$ contact pairs can be found in 226 the $\mathrm{v}(i)-\mathrm{f}(j)$ and $\mathrm{f}(i)-\mathrm{v}(j)$ checking process. The $\mathrm{v}-\mathrm{f}$ checking procedure is shown in $227 \quad$ Fig.7.

228 First, the distance $d_{v f}$ between the vertex coordinate and the infinite plane to which the 229 face belongs is compared to the tolerance $d_{t o l}$. Checking of this pair is continued only 230 if $d_{v f}$ is within the tolerance. If the v-f entrance is valid and $d_{v f}$ is larger than the 
231 tolerance, then blocks $i$ and $j$ cannot contact within the current time step and all

232 checking routines for this block pair are skipped.

233 If $d_{v f}$ is within the tolerance, distance $d_{v f}$ between $\mathrm{v}(i)$ and adjacent faces of $\mathrm{f}(j)$ is

234 checked and the number, $n_{j}$, of v-f pairs that satisfy $d_{v f}<d_{t o l}$ is counted. If distance $d_{v f}$

235 of any v-f pair is larger than the tolerance, proceed to the next pair. Then $n_{j}$ is

236 compared with 1,2 and 3 . If $n_{j} \geq 3$, it is a $\mathrm{V}-\mathrm{v}$ contact pair. If $n_{j}=2$, it is a v-e contact

237 pair. If $n_{j}=1$, it is a v-f contact pair. Then the contact information is calculated and

238 recorded including the VCPs. Only vertices, edges and faces within the VCP region

239 are flagged and detected in following checking procedures.

$240 \quad$ 2.2.2 Edge-to-edge check

241 The flow chart for the crossing e-e contact checking procedure is shown in Fig.8.

242 Orthogonal distance $d_{e e}$ of the two edges is first compared with the contact distance

243 tolerance. Valid entrance check is executed if $\left|d_{e e}\right|>d_{t o l}$, to exclude cases where

244 blocks are close enough but not in contact. If $\left|d_{e e}\right| \leq d_{t o l}$ and the nearest points are

245 within the edge boundaries, the e-e contact information is calculated and recorded. An

246 additional step follows the identification of valid crossing e-e pairs, to compute the

247 VCPs and check if any edge is parallel to adjacent faces of the other edge. If it is, then

248 it is a part of e-f or f-f contact pairs and edges connected with these parallel faces are

249 flagged to be checked.

250 After all v-f and crossing e-e are found, the number $n_{j}$ of contact pairs is counted. If $n_{j}$

251 is 0 , no contacts occur. If $n_{j}$ is 1 , the contact pair belongs to one and only one of $\mathrm{v}-\mathrm{v}$, 
$\mathrm{v}$-e, v-f, crossing e-e types. If $n_{j}$ is 2 , the contact pairs may belong to parallel e-e or e-f

253 contact types. If $n_{j}$ greater than or equal to 3 , the contact pairs belong to f-f contact

254 types. The corresponding $n_{j}$ related elements are then recorded on the contact list.

\subsection{Updated contact detection}

256 The contact types or the contact mode may change from one step to another. However,

257 with the displacement limited by the tolerance, the contact detection results from the

258 previous time step can be used to estimate where contacts may happen, thus reducing

259 the number of contacts that need examinations.

260 In 2-D cases, a contact point on a vertex may move to its adjacent edges and a contact

261 point on an edge may move to its boundary vertex. In 3-D cases, a contact point on a

262 face may move to a boundary edge or a vertex. A contact point on a vertex may move

263 to its connected edges or faces and a contact point on an edge may move to an

264 adjoining vertex or face. Based on the entrance block theory [12], contact covers close

265 to the reference point need to be checked. Based on different contact types, different

266 close objects (vertices, edges, faces) are considered, respectively.

267 The updated checking procedure is shown in Fig.9. It begins by checking whether the

268 neighbor block list changes. If new neighbor blocks are added to the list, the initial

269 contact detection routine is activated. However, for neighbor blocks that existed in the

270 previous time step, the updated contact detection routine is activated according to

271 contact type. First, VCPs are established according to previous contact types, and then

272 distances between the vertices connecting previous contact pairs and the VCP are 
273 checked. The vertices within the VCP tolerance $d_{\text {tol }}$ are counted as a variable $n_{v}$ to

274 track changes of the nearest elements. If $n_{v}$ does not change compared to the previous

275 step, the contact type is retained and a routine to update contact information is

276 followed. If $n_{v}$ changes, the close elements change and an updated detection

277 procedure is followed for new contacting elements. First, a new list of potential

278 checking boundary vertices, edges and faces, is formed. Then $\mathrm{v}(i)-\mathrm{e}(j)$ and $\mathrm{e}(i)-\mathrm{v}(j)$

279 types are checked from the new generated boundary element lists for 2-D polygons,

280 while $\mathrm{v}(i)-\mathrm{f}(j), \mathrm{f}(i)-\mathrm{v}(j)$ and $\mathrm{e}(i)-\mathrm{e}(j)$ types are checked from the new boundary element

281 lists for 3D polyhedra. Basic contact types ( $v-v$ and $v-e$ in 2-D; v-v, v-e, v-f and

282 crossing e-e in 3-D) are obtained through the checking procedure, as well as their

283 contact points, contact normal vectors and normal distance. Then the contact

284 information is recorded in a contact list maintained during the entire calculation 285 process.

\subsection{Data structures and computational cost}

287 The traditional half-edge data structure [18] is used to represent the boundary of a

288 polyhedron. Using this data structure, any geometry element can be obtained quickly.

289 For example, any edges and faces connected to a vertex can be visited using a vertex

290 object; adjacent vertices and faces connected to an edge can be accessed by using an

291 edge object.

292 Assuming polyhedron $i$ has $n_{v i}$ vertices, $n_{e i}$ edges and $n_{f i}$ faces, the calculation cost of

293 the fast direct search algorithm are as follows: In the initial checking subroutine, the 
294 checking cost for two convex polygons is $O\left(n_{v i} \cdot n_{e j}+n_{e i} \cdot n_{v j}\right)$, and $295 O\left(n_{v i} \cdot n_{f j}+n_{f i} \cdot n_{v j}+n_{e i} \cdot n_{e j}\right)$ for two convex polyhedra. During the actual detection 296 computations, the total checking cost may be less because of some exclusion cases: (1)

297 Once a contact pair is found, only adjacent geometry objects are flagged and checked 298 in the subsequent checking procedure; (2) During detection cases of close but 299 not-in-contact neighboring blocks, the exclusion algorithm terminates the unnecessary 300 thorough checking; and (3) During the updated contact detection subroutine, only 301 local boundary areas need to be detected and the computational cost is approximately 302 equal to or less than that of two polygons in the un-optimized procedure.

\section{$303 \quad 3$ Efficiency testing}

\section{$304 \quad 3.1$ Basic 3-D model}

305 The direct search algorithm [8-10], which directly searches v-v, v-e, v-f and crossing 306 e-e contact types, is used as the benchmark in the examples presented herein. A 307 speed-up ratio $R$ is defined as the ratio of the CPU run time for the direct search 308 algorithm to that of the new fast direct search algorithm. Both the initial contact 309 detection procedure and the updated contact detection procedure in the fast direct 310 search algorithm and the fast common plane algorithm [3] are tested.

311 As shown in Fig.10, case 1 examines different contact types (v-v, v-e, v-f, crossing 312 e-e, e-f, f-f and parallel e-e) of two hexahedra and the speed-up ratio is shown in

313 Fig.11. Case 2 considers the different contact types (v-v, v-e, v-f, crossing e-e, e-f, f-f 314 and parallel e-e) of two tetrahedral, as shown in Fig.12. The results are shown in 
315 Fig.13. In Fig.11 and Fig.13 C E-E and P E-E refer to crossing e-e and parallel e-e.

316 Case 3 considers F-F contacts of two blocks that are generated by cutting a

317 hexahedron, as shown in Fig.14. The results are shown in Fig.15.

318 The results show that the speed-up produced by the fast direct search algorithm is

319 significant, although it varies by contact type and shape of the objects in contact. The

320 speed-up variation for the different contact types is related to the numbering sequence

321 of particle topology and the number of adjacent vertices, edges and faces for the

322 contact pair. The average speed-up ratio for different shapes of objects is different; on

323 the order of 3.7 for hexahedrons and on the order of 2.2 for tetrahedra.

324 In basic model test, the FDS algorithm speed-up can be attributed to the following: (1)

325 only v-f and e-e pairs are directly checked, thus reducing computational cost

326 associated with v-v, v-e, e-f and f-f contacts; and (2) After the first contact pair is

327 found, only its adjacent vertices, edges and faces are further examined, thus a

328 thorough v-f and e-e pair checking is avoided.

329 In all basic test cases, the same contact types are obtained by using different contact

330 detection algorithms which illustrates that the method is working correctly. For v-f,

331 crossing e-e, $\mathrm{v}-\mathrm{v}$ (belongs to e-f or $\mathrm{f}-\mathrm{f}$ ) and v-e (belongs to e-f or $\mathrm{f}-\mathrm{f}$ ) case, same

332 contact normal is obtained using DS, FDS and FCP method, with a slightly different

333 contact position, as different contact planes are used (DS and FDS use the face itself

334 as the contact plane while FCP use the common plane as the contact plane). For v-v or

335 v-e that not belong to e-f or f-f types, the contact normals obtained by FDS and FCP 
are slightly different because the different definitions of common plane and virtual

337 contact plane, also the contact position is slightly different owing to the utilization of

338 different contact planes.

\subsection{3-D model evaluation}

340 The performance of the algorithm is evaluated using two test cases, described below.

341 These test cases illustrate how the algorithm performs for densely and sparsely packed

342 particle systems. Additionally, it shows the influence of the number of particles on the

343 algorithm's performance. In both cases, DS, FDS and FCP were used as the contact

344 detection algorithm in the DDA framework to test the detection efficiency, and the

345 detection results of FDS was used to do the simulation. The average time of each

346 delicate contact detection step is used to compute the speed-up ratio of different

347 algorithms. In addition to the comparison of efficiency, the simulation results using

348 FDS algorithm and DDA were also shown to verify the effectiveness of the FDS

349 algorithm.

350 The first case is the stability calculation of a block wall. Three examples were tested,

351 with 15, 55 and 120 blocks, as shown in Fig.16. Each block is assumed rigid and the

352 contact spring stiffness is assumed $1 \mathrm{E} 10 \mathrm{~N} / \mathrm{m}$. Initial time step is set as $0.001 \mathrm{~s}$ and the

353 contact detection times for the first 1000 time steps are presented for comparison, as

354 shown in Table 1. During the computation, the vertical displacements of the top block

355 centroids in the three examples are recorded, as shown in Fig.17. In this case, blocks

356 are densely packed with a large number of contact pairs. The results show that the 

gradually converge.

360 The second case involves loosely distributed particles falling due to gravity. Three

361 models were tested, with 64, 125 and 216 blocks, as shown in Fig.18. All particles are

362 assumed rigid tetrahedral blocks, with volumes around $1.7 \mathrm{E}-4 \mathrm{~m}^{3}$, and the contact

363 spring stiffness is assumed as $1 \mathrm{E} 5 \mathrm{~N} / \mathrm{m}$. The initial time step is $0.001 \mathrm{~s}$ and the contact

364 detection times for the first 3000 time steps are presented for comparison, as shown in

365 Table 1. In this case, there are many neighbor block pairs that may not be in contact

366 with each other. The results show that the speed-up ratio is quite significant, by a

367 factor between 5 and 6 for FDS and on the order of 3+ for FCP. This speed-up of FDS

368 and FCP compared to DS is principally owing to the reduction of the cost of detecting

369 contact pairs of not-in-contact neighboring blocks.

\section{Conclusions and discussions}

371 We present an improved contact detection algorithm for convex polyhedra, by using

372 initial contact detection and updated contact detection in a serial analysis. This

373 improved algorithm has several benefits. First, the contact detection is more efficient

374 because the updated contact detection algorithm reuses the results from the prior time

375 step. Second, thorough examination of contact pairs is avoided owing to the

376 consideration of spatial relationships, properties of convex particles, and the

377 assumption of small displacement in a time step. Third, the exclusion subroutine 
378 increases the efficiency of the detection algorithm because it avoids checking of

379 contact pairs with the least possible overhead. The test results show that the fast direct

380 search algorithm produces respectable speed-up in evaluating contact between

381 tetrahedra and hexahedra. To further improve the efficiency of contact detection, this

382 algorithm can be extended using parallel computation techniques.

\section{Acknowledgement}

384 This study is supported by the National Basic Research Program of China (973

385 Program) (2014CB046904, 2014CB047101) and the China National Natural Science

386 Foundation (51479191). The first author is supported by the China Scholarship

387 Council during his study at UC Berkeley. The third and fourth authors are supported

388 in part by the National Science Foundation (NSF) grant CMMI-1363354 and the

389 Edward G. Cahill and John R. Cahill Endowed Chair funds.

390 References

391 [1] Boon CW, Houlsby GT, Utili S. A new algorithm for contact detection between

392 convex polygonal and polyhedral particles in the discrete element method.

$393 \quad$ Comput Geotech 2012; 44: 73-82

394 [2] Cundall PA. Formulation of a three-dimensional distinct element model-Part I. A

395 scheme to detect and represent contacts in a system composed of many

396 polyhedral blocks. Int J Rock Mech Min Sci \& Geomech Abstr 1988; 25 :

$397 \quad 107-116$ 
398 [3] Nezami EG, Hashash YMA, Zhao D, Ghaboussi J. A Fast Contact Detection

$399 \quad$ Algorithm for 3-D Discrete Element Method. Comput Geotech 2004; 31 :

$400 \quad 575-587$

401 [4] Nezami EG, Hashash YMA, Zhao D, Ghaboussi J. Shortest link method for

402 contact detection in discrete element method. Int J Numer Anal Meth Geomech

$403 \quad 2006 ; 30: 783-801$

404 [5] Liu XL, Lemos JV. Procedure for Contact Detection in Discrete Element Analysis.

$405 \quad$ Adv Eng Softw 2001; 32: 409-415.

406 [6] Keneti AR, Jafari A, Wu JH. A New Algorithm to Identify Contact Patterns

407 between Convex Blocks for Three-Dimensional Discontinuous Deformation

$408 \quad$ Analysis. Comput Geotech 2008; 35: 746-759

409 [7] Wu JH, Juang CH, Lin HM. Vertex-to-face contact searching algorithm for

$410 \quad$ three-dimensional frictionless contact problems. Int J Numer Meth Eng 2005;

$411 \quad 63(6): 876-897$

412 [8] Zhang H, Chen GQ, Zheng L, Han Z, Zhang YB, Wu YQ, et al. Detection of

413 contacts between three-dimensional polyhedral blocks for discontinuous

414 deformation analysis. Int J Rock Mech Min Sci 2015; 78: 57-73

415 [9] Zheng F, Jiao YY, Zhang XL, Tan F, Wang L, Zhao Q. Object-Oriented Contact

416 Detection Approach for Three-Dimensional Discontinuous Deformation

417 Analysis Based on Entrance Block Theory. Int J Geomech 2016; E4016009. 
418 [10] Zhang H, Liu SG, Han Z, Zheng L, Zhang YB, Wu YQ, et al. A new algorithm

419 to identify contact types between arbitrarily shaped polyhedral blocks for 420 three-dimensional discontinuous deformation analysis. Comput Geotech 2016; $421 \quad 80: 1-15$

422 [11] Wu W, Zhu HH, Zhuang XY, Ma GW, Cai YC. A multi-shell cover algorithm 423 for contact detection in the three dimensional discontinuous deformation analysis. Theor Appl Fract Mec 2014; 72: 136-149

425 [12] Shi GH. Contact Theory. Sci China Tech Sci 2015; 58: 1-47.

426 [13] Munjiza A, Andrews KRF. NBS contact detection algorithm for bodies of similar size. Int J Numer Meth Eng 1998; 43(1): 131-149

428 [14] Perkins E, Williams JR. A fast contact detection algorithm insensitive to object sizes. Eng Computation 2001; 18: 48-62

430 [15] Williams JR, Perkins E, Cook B. A contact algorithm for partitioning N arbitrary $431 \quad$ sized objects. Eng Computation 2004; 21: 235 - 248

432 [16] Shi GH. Discontinuous deformation analysis: a new numerical model for the 433 statics and dynamics of block systems. Ph.D. Thesis. University of California, $434 \quad$ Berkeley; 1998.

435 [17] Muller DE, Preparata FP. Finding the Intersection of Two Convex Polyhedra. 436 Theor Comput Sci 1978; 7: 217-236 


\section{Table list}

Table 1 Speed-up ratios of FDS and FCP

\begin{tabular}{ccccc}
\hline Examples & Block Number & Time Steps & FDS & FCP \\
\hline Block wall & 15 & 1000 & 4.97 & 2.48 \\
case & 55 & 1000 & 4.98 & 2.48 \\
\hline Dropping & 120 & 1000 & 4.97 & 2.46 \\
particle case & 64 & 3000 & 5.19 & 3.29 \\
& 125 & 3000 & 5.35 & 3.70 \\
\hline
\end{tabular}




\section{Figure Caption list}

443 Fig. 1. Entrance of vertex-to-edge and its VCPs.

444 Fig. 2. Entrance of vertex-to-face and its VCPs.

445 Fig. 3. Entrance of crossing edge-to-edge and its VCPs in 3D polyhedra.

446 Fig. 4. Entrance of vertex-to-vertex and its VCPs in 2D polygons.

447 Fig. 5. Initial v-e checking procedure for convex polygons.

448 Fig. 6. Initial contact checking procedure for convex polyhedra.

449 Fig. 7. Initial v-f checking procedure for convex polyhedra.

$450 \quad$ Fig. 8. Initial e-e checking procedure for convex polyhedra.

451 Fig. 9. Updated contact checking procedure for convex polyhedra.

452 Fig. 10. Different contact types of two hexahedra.

453 Fig. 11. Speed-up ratios of FDS and FCP for two hexahedra.

454 Fig. 12. Different contact types of two tetrahedra.

455 Fig. 13. Speed-up ratios of FDS and FCP for two tetrahedra.

456 Fig. 14. Different f-f contact cases between two polyhedra generated by block cutting.

457 Fig. 15. Speed-up ratios of FDS and FCP for two polyhedra.

458 Fig. 16. Simulation of block wall models: (a) model 1 with 15 blocks; (b) model 2 459 with 55 blocks; (c) model 3 with 125 blocks.

460 Fig. 17. Accumulated vertical displacement of measured points using FDS algorithm.

461 Fig. 18. Simulation of dropping particle models using FDS algorithm: (a) model 1 462 with 64 blocks; (b) model 1 after 3000 steps; (c) model 2 with 125 blocks; (d) model 4632 after 3000 steps; (e) model 3 with 216 blocks; (f) model 3 after 3000 steps. 
464 Fig 1 Entrance of vertex-to-edge and its VCPs.

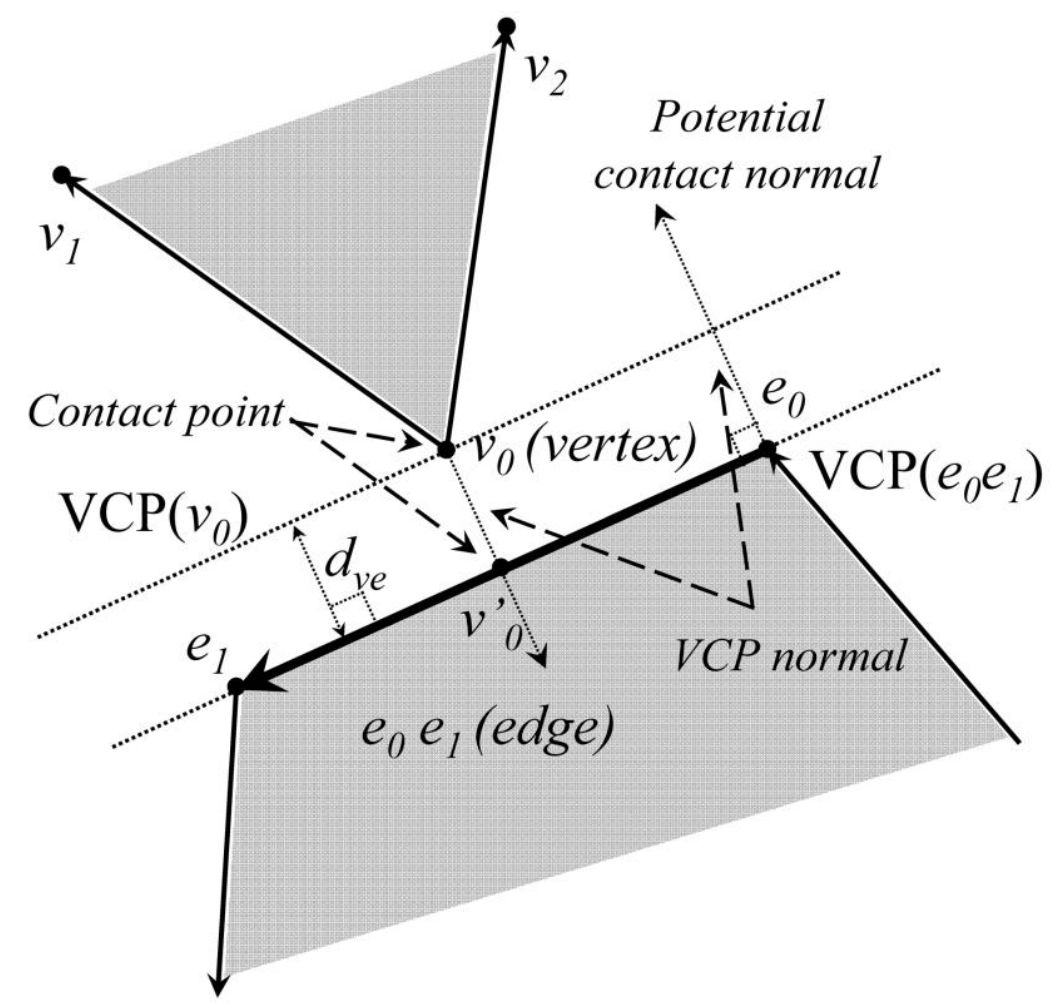


466 Fig 2 Entrance of vertex-to-face and its VCPs.

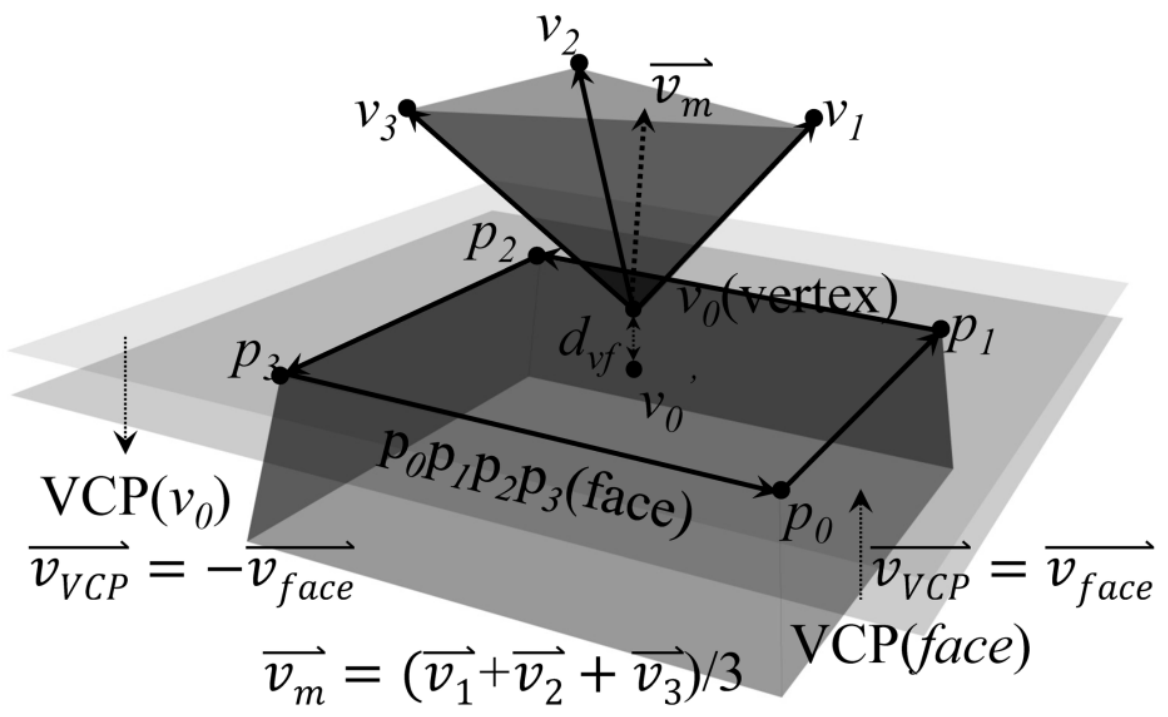


468 Fig 3 Entrance of crossing edge-to-edge and its VCPs in 3D polyhedra.

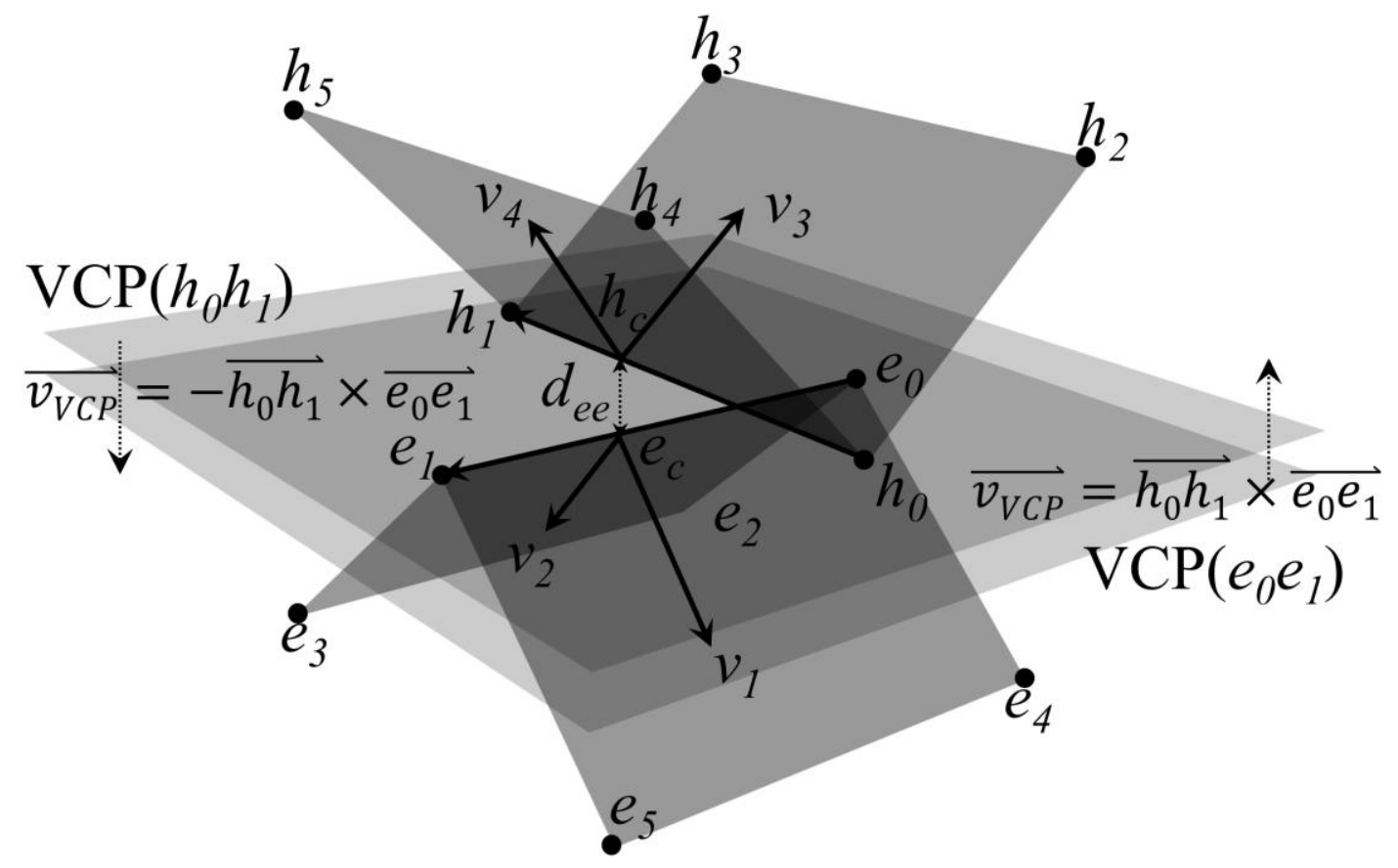


470 Fig 4 Entrance of vertex-to-vertex and its VCPs in 2D polygons.

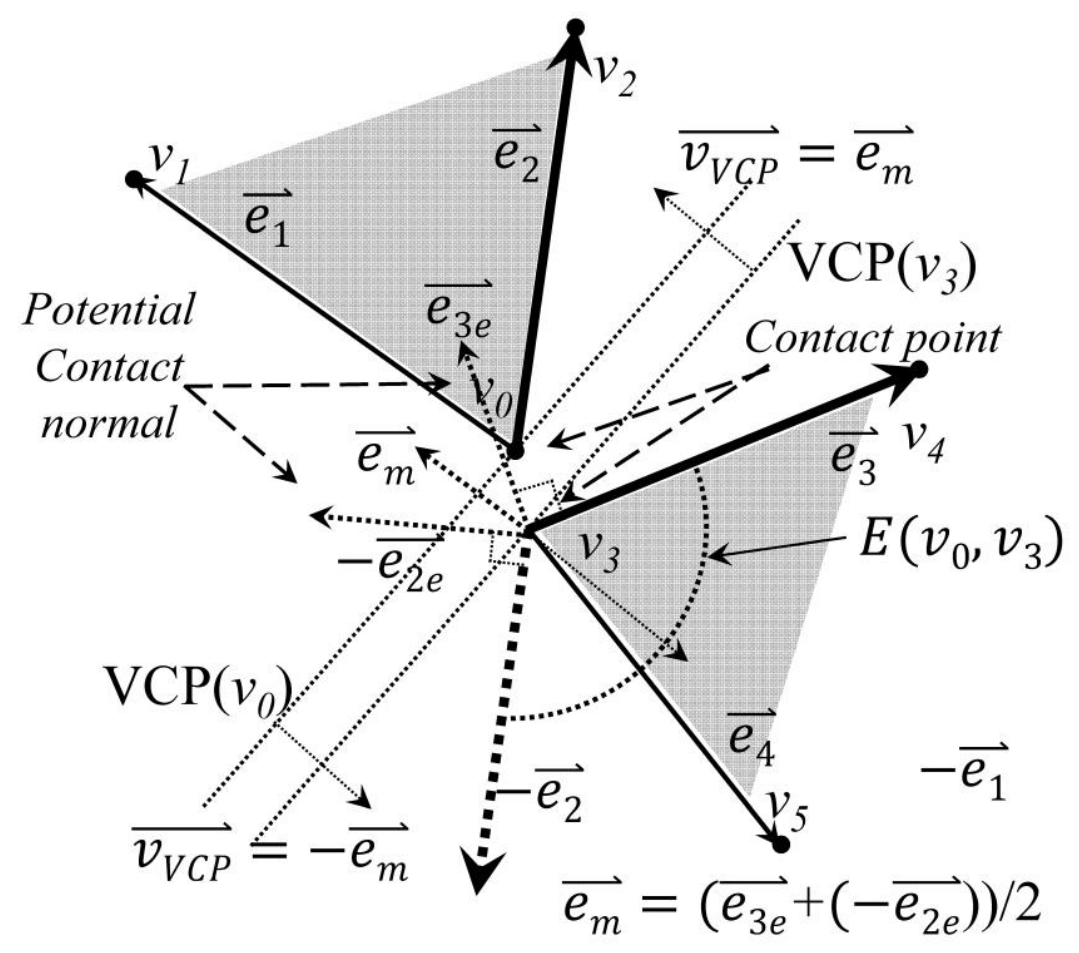


472 Fig 5 Initial v-e checking procedure for convex polygons.

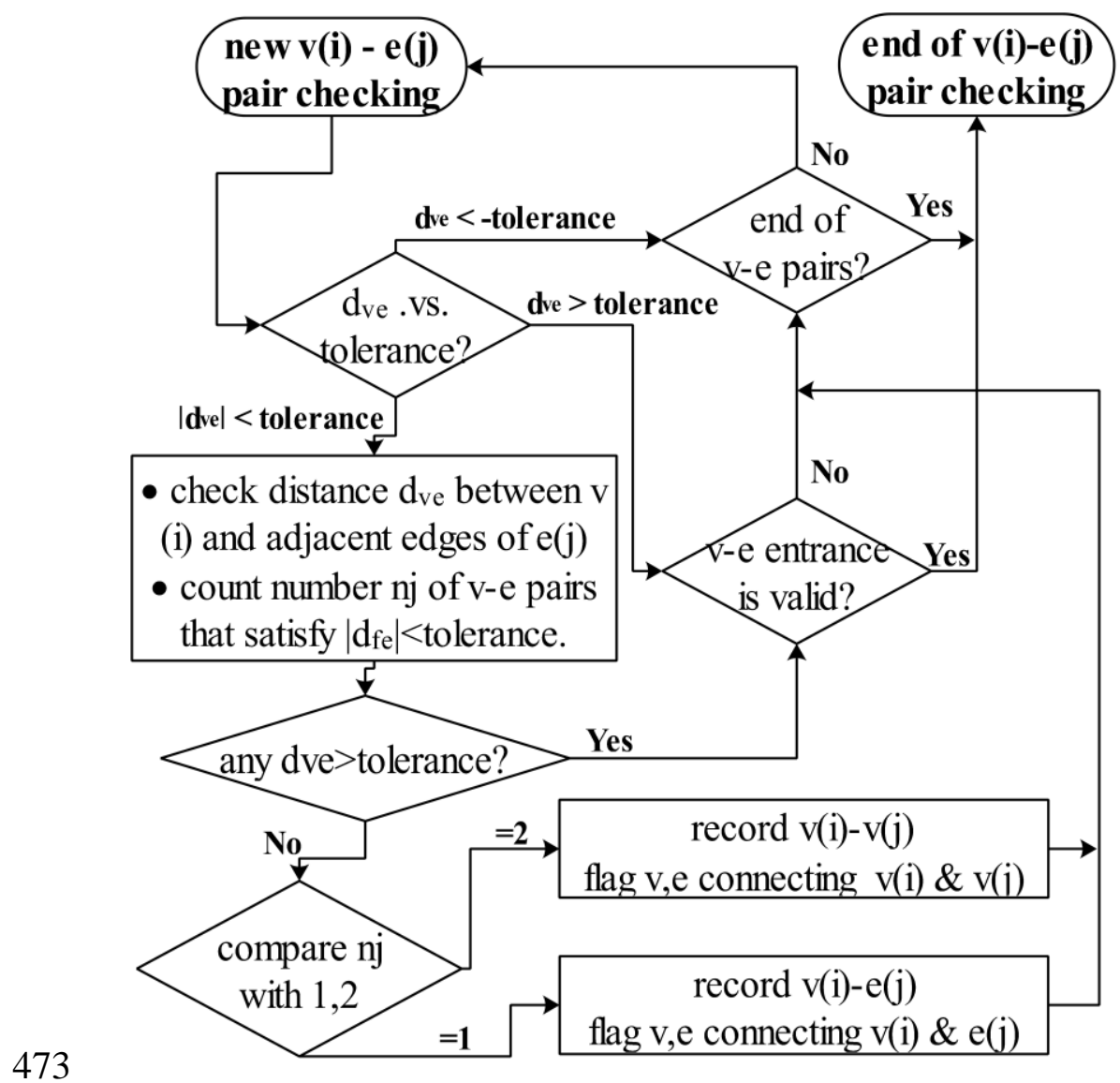


474 Fig 6 Initial contact checking procedure for convex polyhedra.

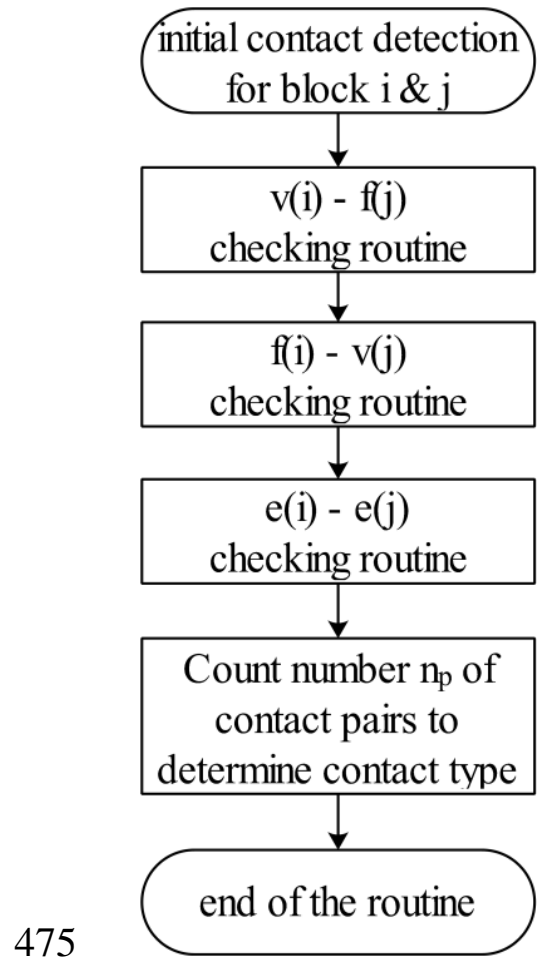


476 Fig 7 Initial v-f checking procedure for convex polyhedra.

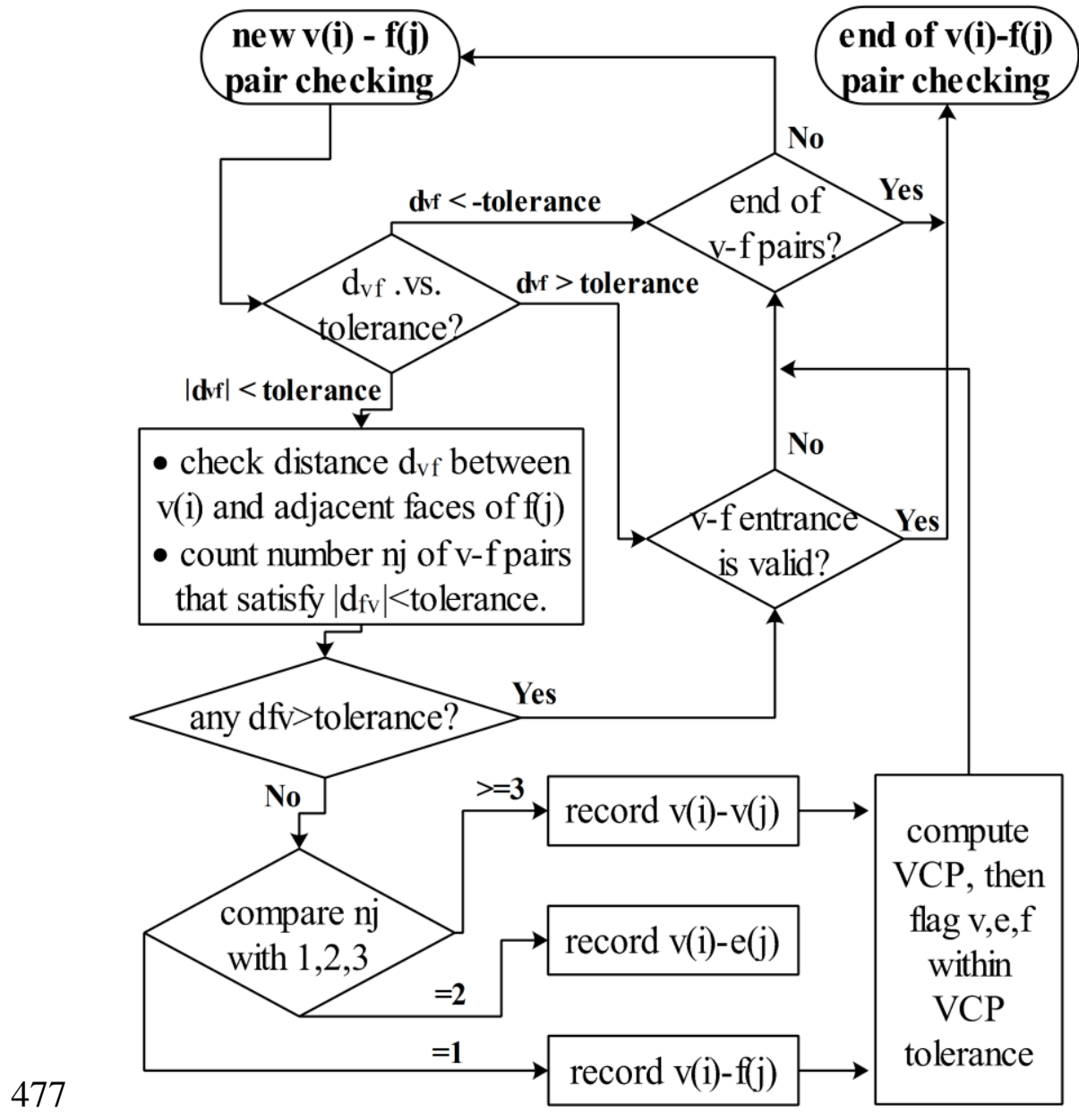


478 Fig 8 Initial e-e checking procedure for convex polyhedra.

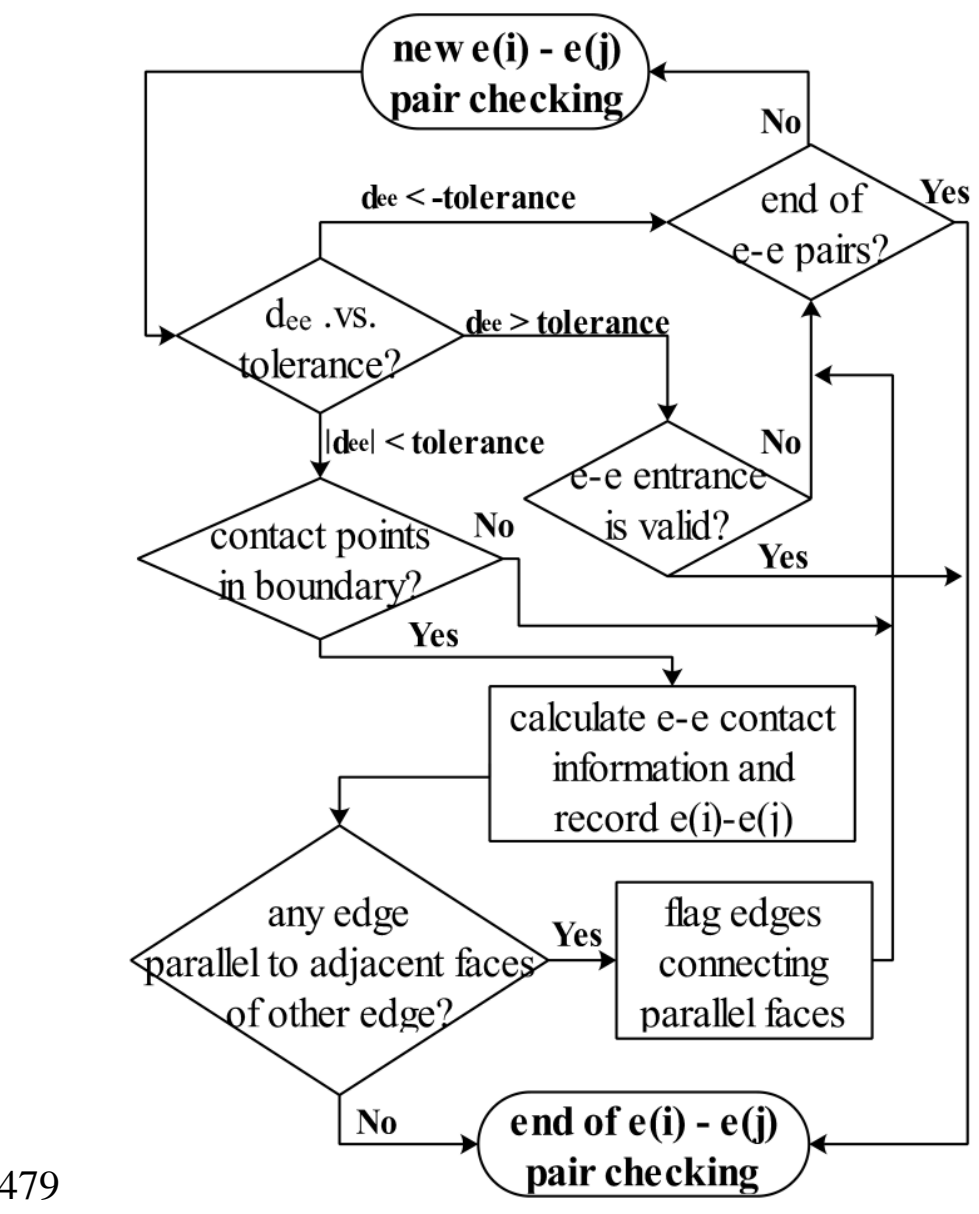


Fig 9 Updated contact checking procedure for convex polyhedra.

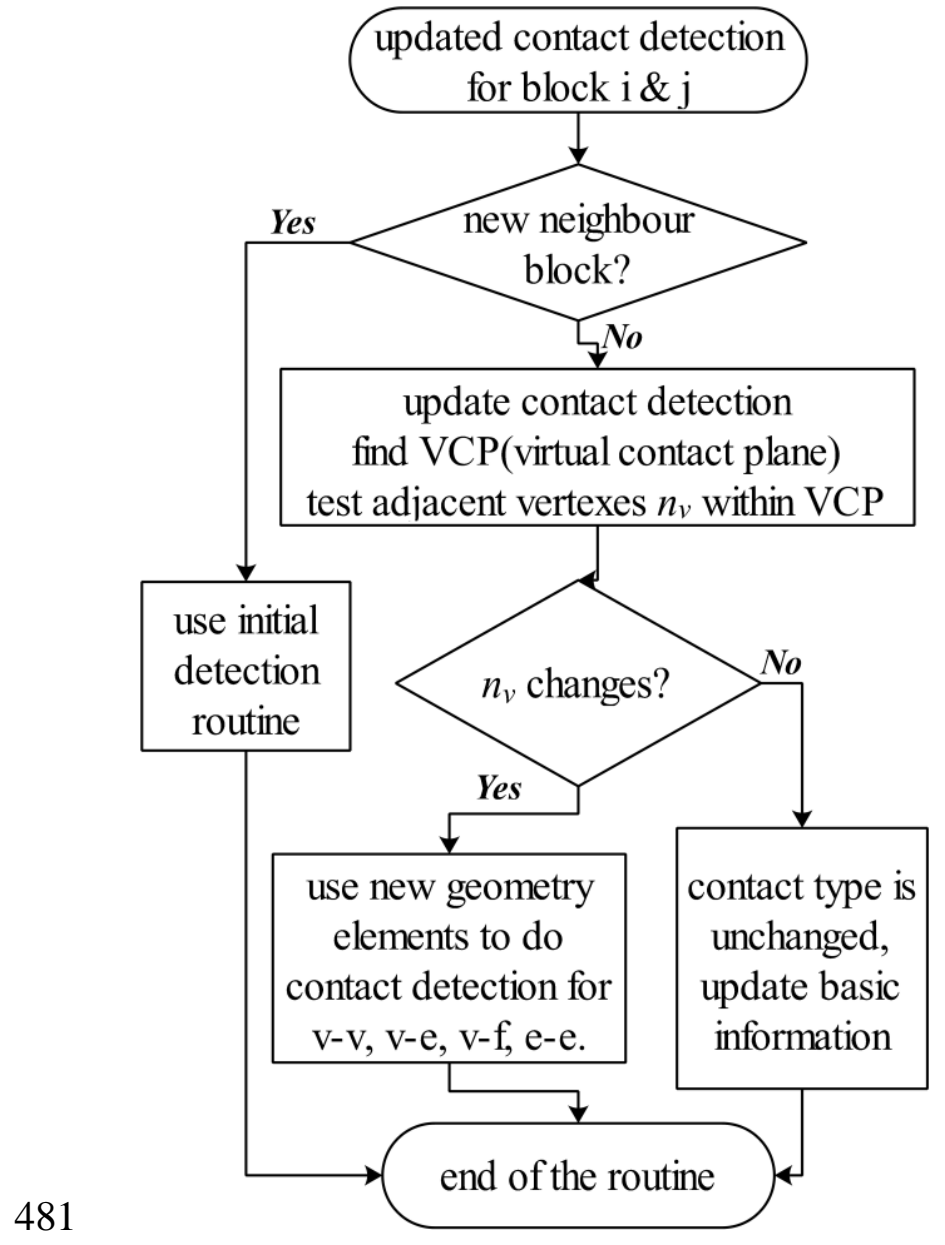


482 Fig 10 Different contact types of two hexahedra.

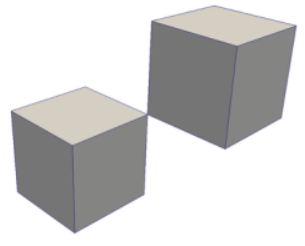

V-V

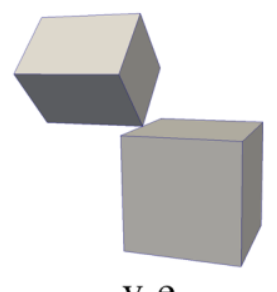

V-e

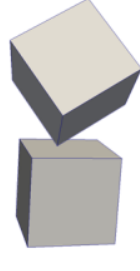

$v-f$

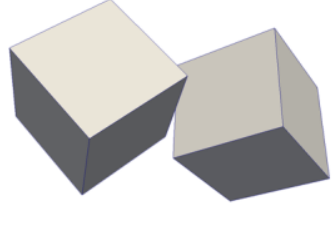

crossing e-e

483
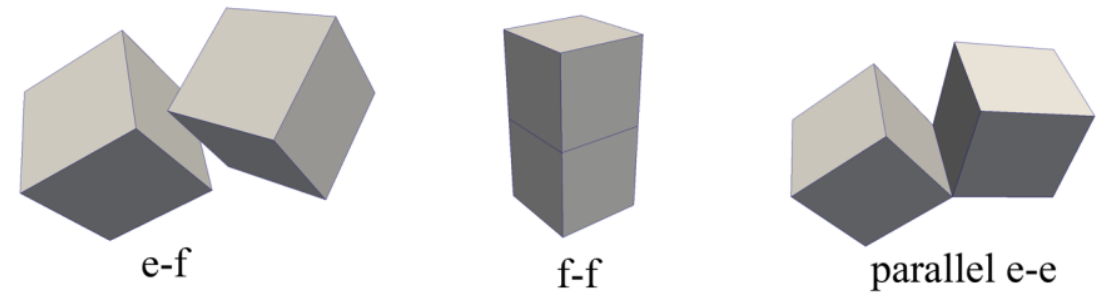
484 Fig 11 Speed-up ratios of FDS and FCP for two hexahedra.

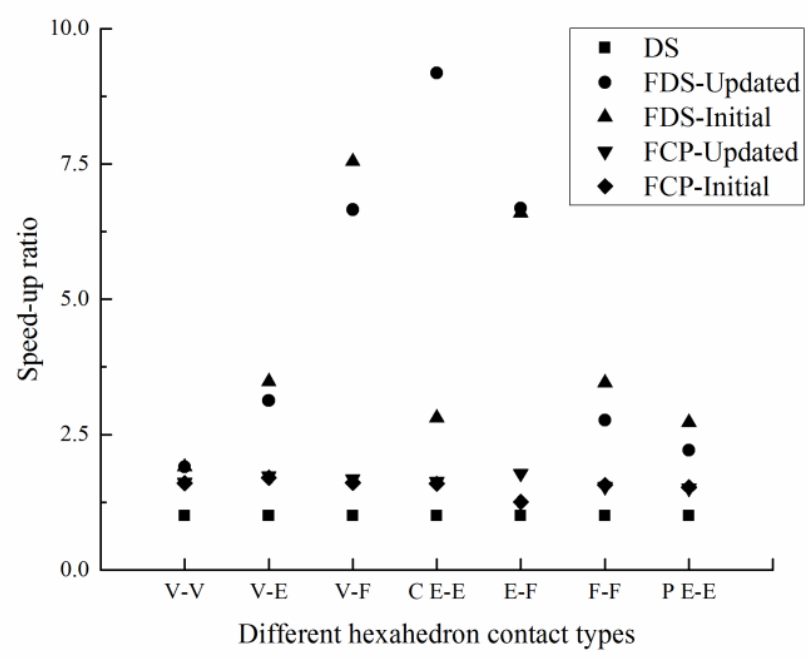


486 Fig 12 Different contact types of two tetrahedra.

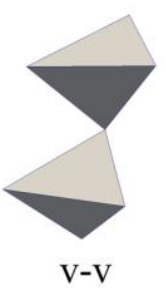

87

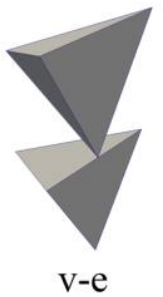

v-e
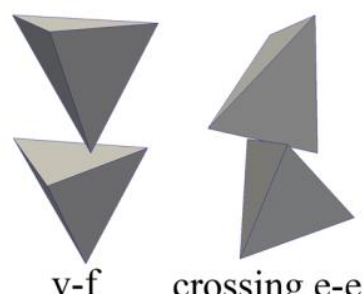

crossing e-e

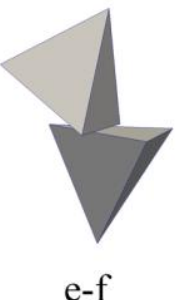

e-f

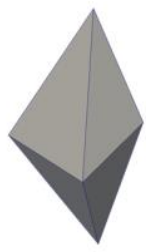

f-f parallel e-e

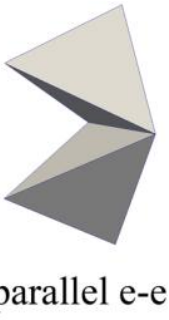


488 Fig 13 Speed-up ratios of FDS and FCP for two tetrahedra.

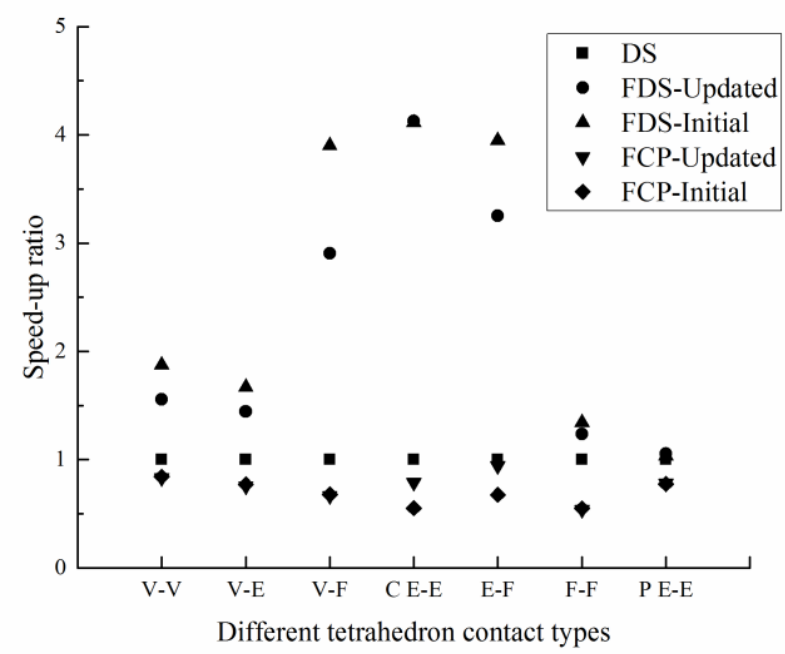


490 Fig 14 Different $\mathrm{f}-\mathrm{f}$ contact cases between two polyhedra generated by block cutting.

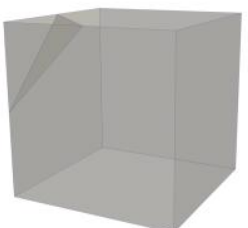

f-f 1

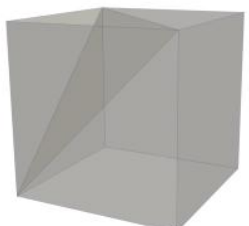

f-f 2

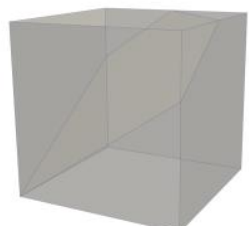

f-f 3

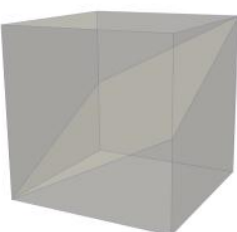

f-f 4

491 
492 Fig 15 Speed-up ratios of FDS and FCP for two polyhedra.

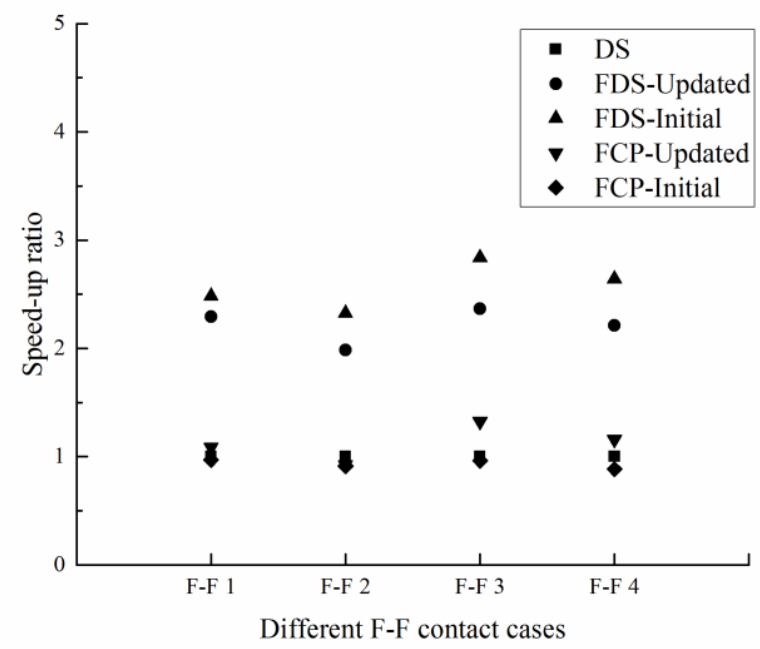

493 
494 Fig. 16. Simulation of block wall models:

495 Fig 16 (a) model 1 with 15 blocks;

496

497 Fig 16 (b) model 2 with 55 blocks;

498

499 Fig 16 (c) model 3 with 125 blocks. 
501 Fig 17 Accumulated vertical displacement of measured points using FDS algorithm.

Accumulated Vertical displacement of measured points

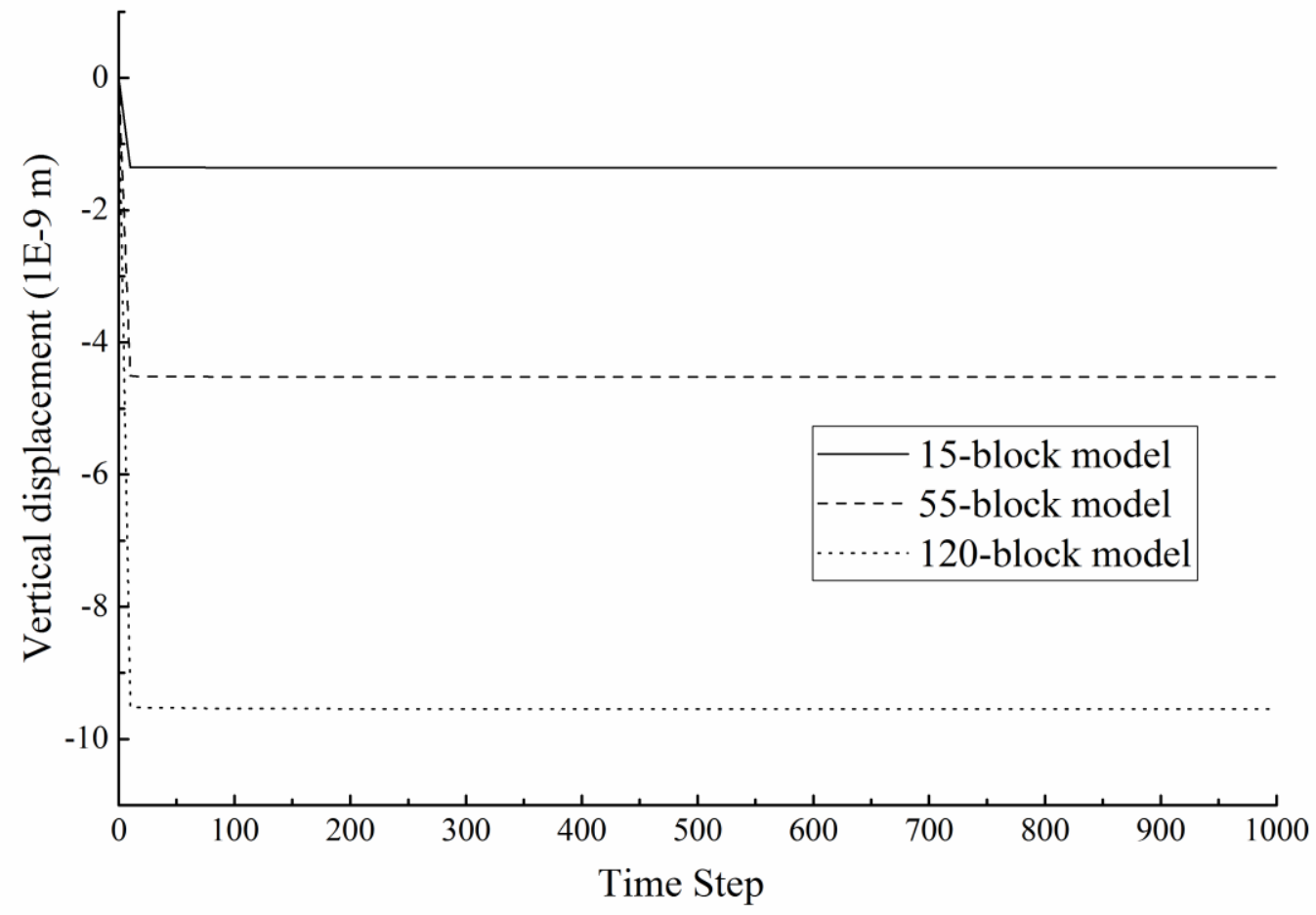

502 
503 Fig. 18. Simulation of dropping particle models using FDS algorithm:

504 Fig 18 (a) model 1 with 64 blocks;

505

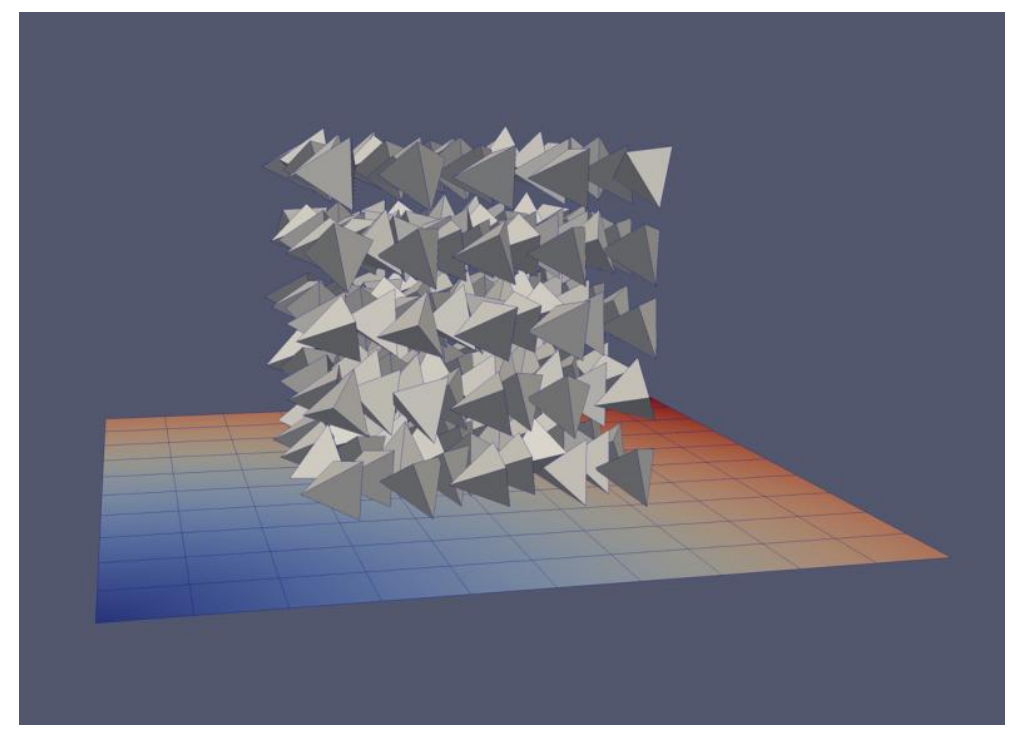

506 Fig 18 (b) model 1 after 3000 steps;

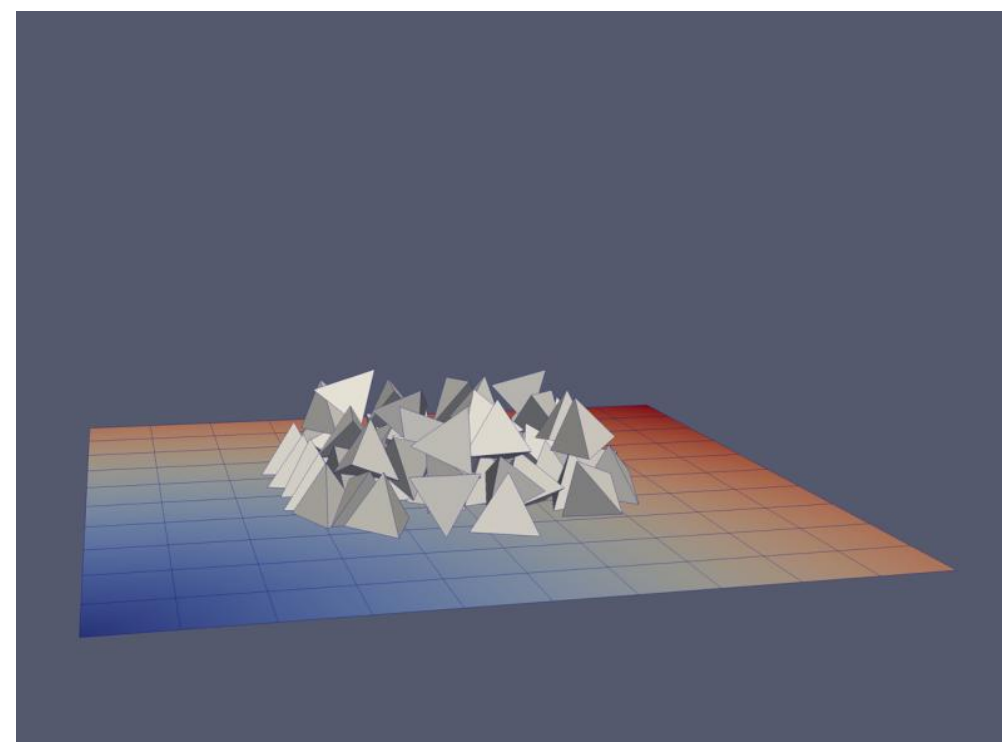


509 Fig 18 (c) model 2 with 125 blocks;

511 Fig 18 (d) model 2 after 3000 steps;

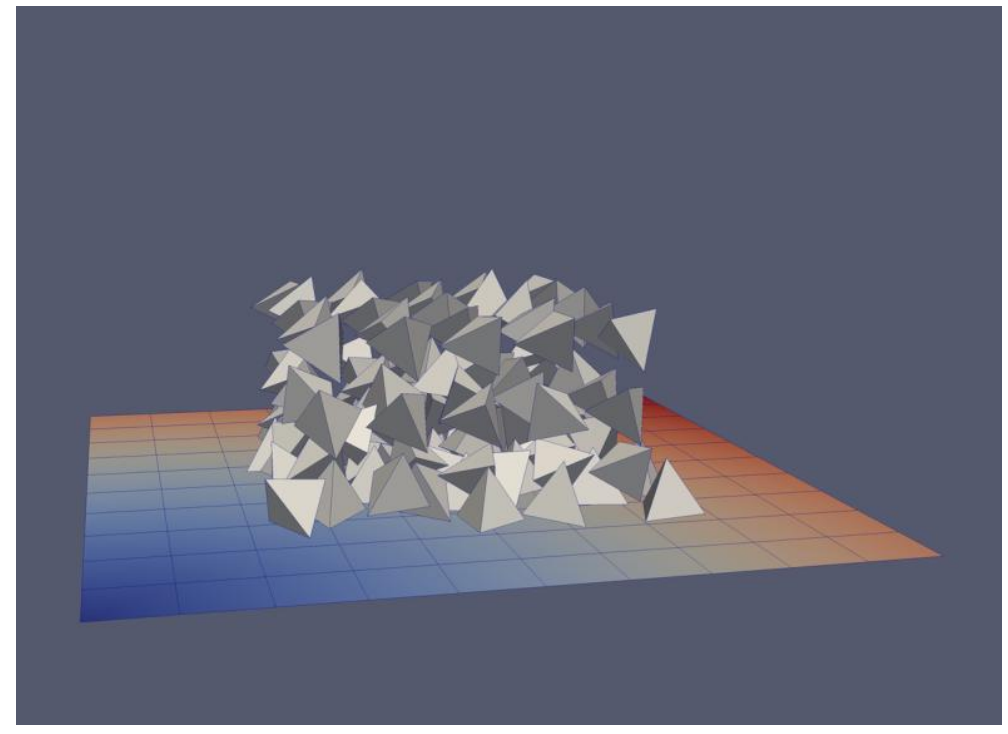

512 
514 Fig 18 (e) model 3 with 216 blocks;

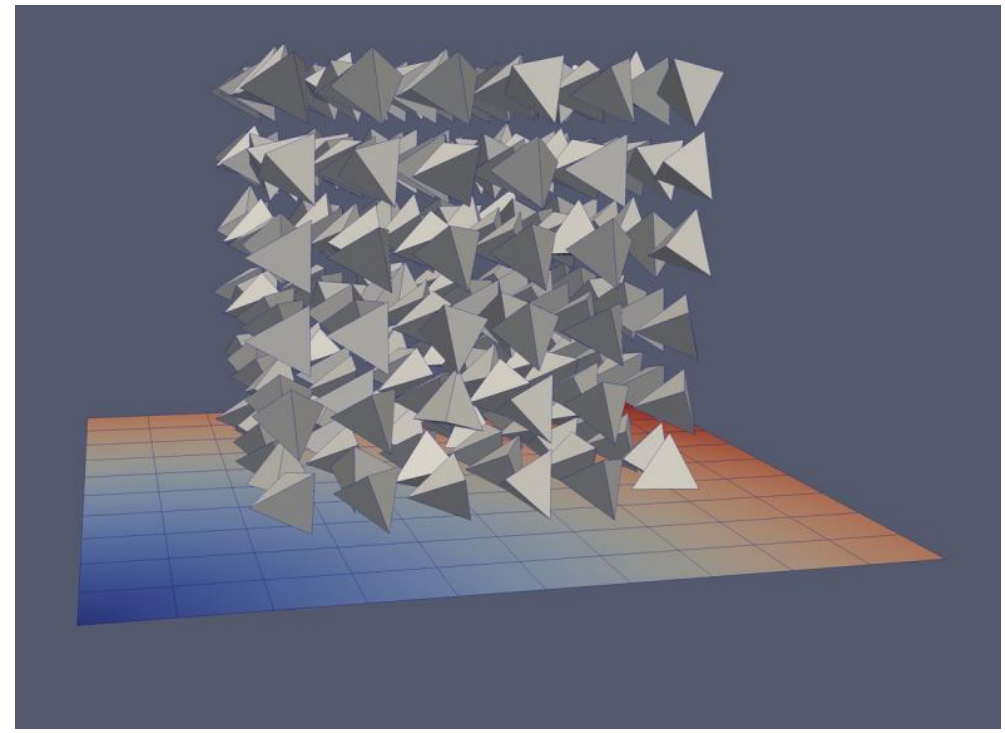

515

516 Fig 18 (f) model 3 after 3000 steps.

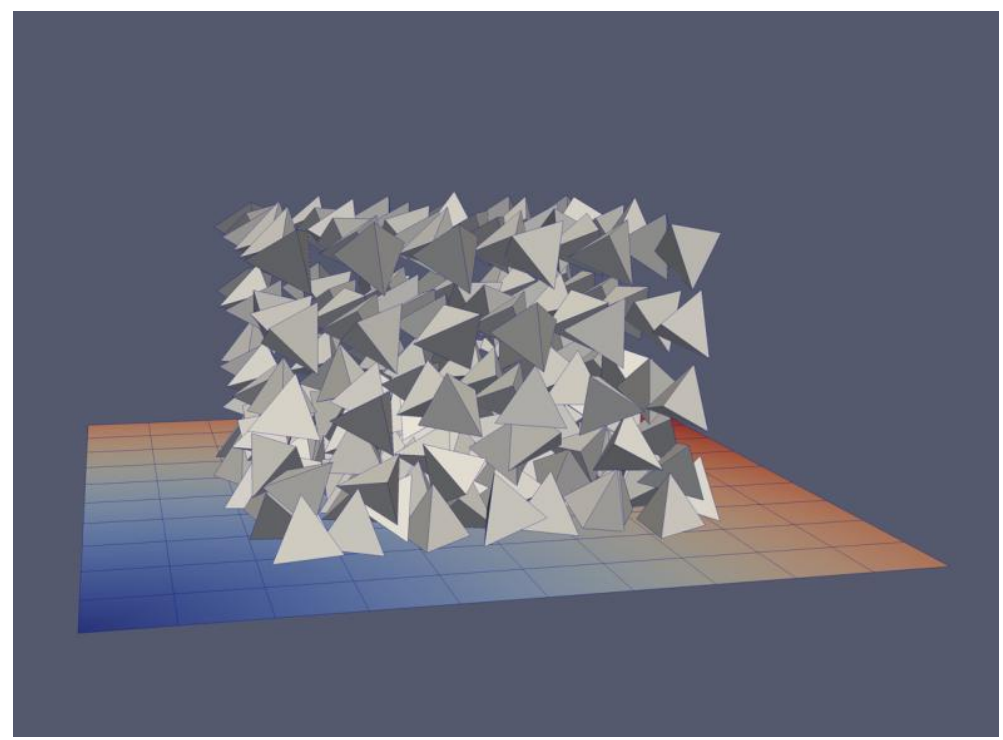

